Article

\title{
Workplace Ostracism and Knowledge Hiding: The Mediating Role of Job Tension
}

\author{
Sidra $\operatorname{Riaz}^{1, *(D)}$, Yusen $X u^{1,2, *,+}$ and Shahid Hussain ${ }^{3}(D)$ \\ 1 Faculty of Management and Economics, Dalian University of Technology, Dalian 116024, China \\ 2 School of Business, Shantou University, Shantou 515063, China \\ 3 Department of Mathematics, COMSATS University Islamabad, Attock Campus 43600, Pakistan; \\ shahidhussain@cuiatk.edu.pk \\ * Correspondence: riaz_sidra@yahoo.com (S.R.); ipmp@dlut.edu.cn (Y.X.) \\ + Affiliation 2 is the main affiliation for Yusen $\mathrm{Xu}$.
}

Received: 10 July 2019; Accepted: 22 September 2019; Published: 9 October 2019

\begin{abstract}
This study examined the impact of workplace ostracism on employees' knowledge hiding behavior. Based on the conservation of resource theory, this study seeks to identify the effects of job tension as a mediator and the moderating effect of employee loyalty. Using a time-lagged research design, we collected the data from 392 employees of the textile industry. Results indicate that workplace ostracism positively influences the knowledge hiding behavior such as evasive hiding and playing dumb, whereas it is not significantly related with rationalized hiding. More specifically, workplace ostracism increases the feeling of job tension, while job tension mediates the relationship between workplace ostracism and knowledge hiding. In addition, we found that workplace ostracism demolished the benefits of employee loyalty, as high workplace ostracism more strongly influences the feeling of job tension. We also discussed the theoretical and practical implications.
\end{abstract}

Keywords: workplace ostracism; knowledge hiding; evasive hiding; playing dumb; rationalized hiding; job tension; employee loyalty

\section{Introduction}

The growing importance of knowledge sharing has emerged as one of the key steps in organizational success. Many scholars highlighted that such phenomenon is crucial for production companies to promote innovation and improve performance [1,2]. Although these studies encourage knowledge sharing, Connelly, et al. [3] noted that employees choose not to provide access to their knowledge, defined as knowledge hiding. Which refers to the "intentional attempt by an individual to withhold or conceal the requested knowledge" [3]. Prior studies considered the knowledge hiding as detrimental to organizational performance, compared with its negative effects the factors that predict it have not been extensively studied [3,4].

Recent research by Zhao, et al. [4] pointed out that knowledge hiding is closely related with the process of social exchange and reciprocal beliefs, which extends the tendency of negative response to negative treatment among members at workplace. However, the social exchange among employees depends on workplace climate and mutual trust [5]. In the organizational context, workplace ostracism is considered as a major factor that can eradicate social exchange behavior. Current research examined the negative effects of workplace ostracism on in-role behavior and organizational citizenship behavior $[5,6]$. Workplace ostracism as a pervasive phenomenon can reduce the employee work engagement and resulting performance of the organization. Literature shows that workplace ostracism can attenuate the helping behavior and enhance counterproductive work behavior [7]. However, Zhao, et al. [4] reported 
that workplace ostracism negatively affects the employees' pro-social behavior. Therefore, it appears that as a work stressor it may be a key predictor of knowledge hiding.

Recent research investigated workplace ostracism beyond the direct effects and found mediating variables such as organizational identification, stress, person-organization fit (e.g., [8-10]). For instance, Ferris, et al. [8] reported that global self-esteem mediates the relationship among workplace ostracism and job performance, Zhao, et al. [4] indicated that negative reciprocity beliefs and moral disengagement enhance knowledge hiding behavior as a result of workplace ostracism. However, to better understand the conditions under which the relationship between knowledge hiding and workplace ostracism would be more strengthened or weakened, recent research of Zhao, et al. [4] suggested that job context would play an important role in employees' reaction to ostracism [11]. This study sheds light on the mediating mechanisms between workplace ostracism and knowledge hiding by suggesting job tension as a major mediator. Job tension can act as a mediator because it reflects the impact of stressors at workplace [3]. In this regard, Zhao, et al. [4] found that job tension mediates the relationship between workplace ostracism and employees' counterproductive work behaviors. However, the conservation of resources (COR) theory suggests that when individuals are threatened with resource loss they become determined to retain their threatened resources, such strain may impair individuals' capacity to fulfill job demands. Thus, this study contributes to extend the literature through empirically investigating the mediating effect of job tension among workplace ostracism and knowledge hiding behavior.

In addition to the relationship between workplace ostracism and job tension, we further investigate the boundary effects under which such relation is more or less likely to occur. Although workplace ostracism effects are pervasive, prior research shows that coping responses vary among individuals [12], more stress and tension may develop among individuals who have a high level of loyalty toward an organization [11]. Based on prior research, we predict that employees with a high level of loyalty are more likely to feel job tension $[13,14]$.

In addressing the above purposes, the major contribution of this study is to empirically explore the field of workplace ostracism and knowledge hiding literature. First, it enriches the hiding knowledge literature (i.e., $[3,15,16])$ by identifying workplace ostracism as an antecedent of psychological stress that depletes participative behavior. Second, based on the COR theory, it extends the boundary conditions under which workplace ostracism through job stress influences knowledge hiding. Third, by examining the moderating effect of employee loyalty, employee loyalty as an assurance of employees' useful and positive contributions increases the performance and efforts but this study elaborates that it can also enhance negative effects. Lastly, in view of the critical role of employees' feelings for an organization [17], these results provide an insight into minimizing the occurrence of workplace ostracism to help minimize the knowledge hiding behavior and increase the organizational effectiveness. Figure 1 provides an overview of our theoretical framework. 


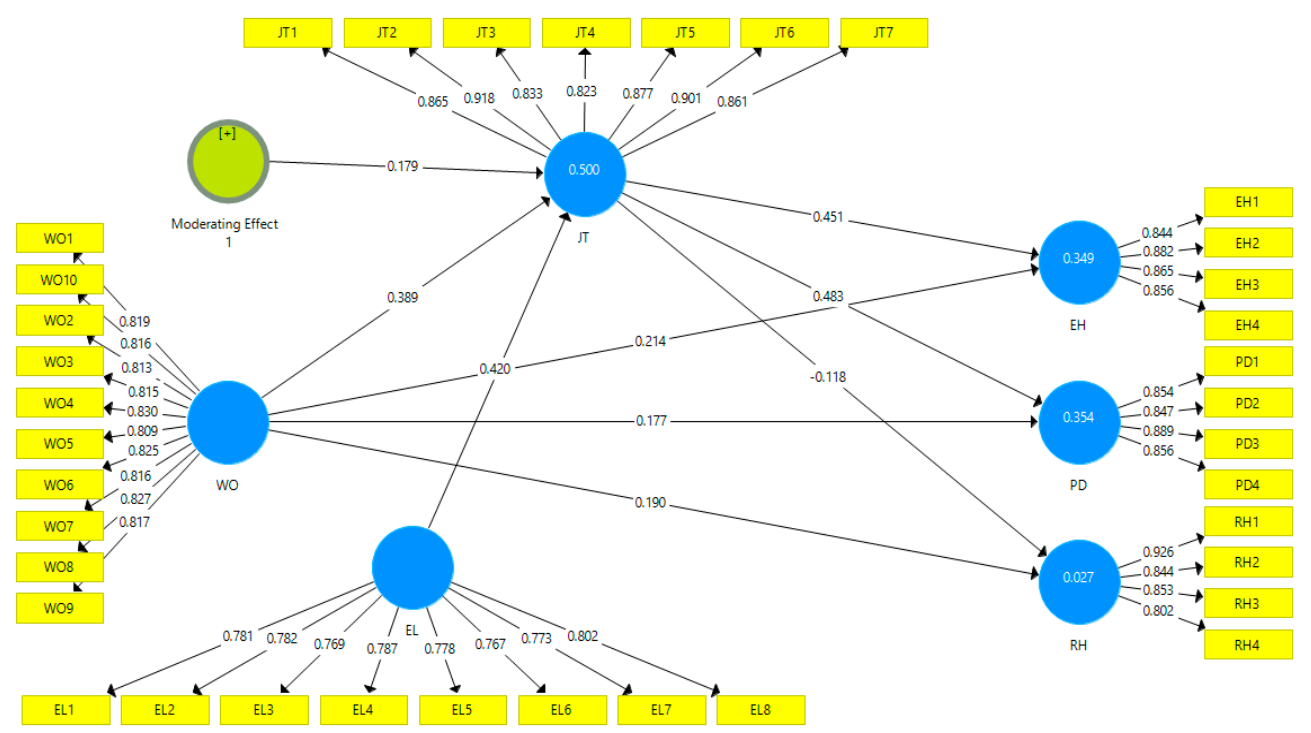

Figure 1. Measurement model with factor loadings.

\section{Literature Review and Conceptual Framework}

\subsection{Knowledge Hiding}

When employees exchange their tacit and explicit knowledge with their co-workers, it contributes to the overall performance, innovativeness, and competency of an organization $[18,19]$. While knowledge hiding is intentional in nature, employees may refuse to share it with another person [3]. This knowledge encompasses information relevant to some tasks or job expertise (e.g., [2]). The intentional refusal of knowledge sharing reflects the distinction between knowledge hoarding and hiding [20]. Therefore, it has dire consequences on knowledge sharing and organizational performance. However, according to Connelly, et al. [3] this concept is also not same as CWBs because knowledge hiding is not necessarily intended to harm other people and organization, while CWBs are a set of volitional acts that intend to harm others or their organization. However, knowledge hiding may have some positive intentions. For example, rationalized hiding may be intended to protect the other's feeling or preserve confidentiality (e.g., refusal to share some confidential documents). Knowledge hiding is dyadic and cannot be considered as uniformly negative behavior while CWBs may be intended to harm organizations or stakeholders $[3,11]$.

To conceal knowledge, employees use three strategies and the adoption of strategy depends on the type of knowledge question [3]. Employees will tend to adopt evasive hiding if the nature of requested knowledge is complex. According to [15], evasive hiding occurs when "the hider provides incorrect information or misleading promise of a complete answer in the future" (p. 480). It involves deception, even though an employee has no intention to provide information. The second strategy can be defined as "feigning ignorance of the requested knowledge" [21] p. 3. For example, an employee pretends not to have the requested knowledge. And the third strategy is called rationalized hiding which involves "offering a justification by giving reasons or blaming someone else" [21] p. 3. It is used in the case of confidential information and also in order to protect co-workers' feelings. Although an individual may consider knowledge hiding intentions as positive, these exert a negative impact on organizational performance, corporation, and development [16]. However, individual willingness factor to conceal knowledge has not been fully explored. Such concealment still occurs, even though organizational practices are designed to facilitate knowledge sharing [3,22].

\subsection{Workplace Ostracism}

The practice of social exclusion, interpersonal rejection, mistreatment and ostracism have become the focus of organizational studies $[7,11]$. It leads to numerous negative outcomes at both organizational and individual level because it causes a sense of social pain [23]. Ostracism is defined as "the extent to 
which an individual perceives that he or she is ignored or excluded by others at workplace" [24] p. 1348. It threatens the basic needs of self-esteem, need of belonging, need of control, and need for meaningful existence. Previous studies that investigated workplace ostracism found that it can perpetrated by any member of an organization, including supervisors, in-group or out-group co-worker or customer. However, the perception of being ostracized is subjective. The person who is ostracized at work can engage in both subtle and retaliatory behaviors against his/her coworkers $[8,11]$. Whereas these negative outcomes also affect individual's ability to self-regulate, they also affect his/her own health, decrease job satisfaction, and civic behavior [25]. However, many studies focused on this topic and found its impact on interpersonal behavior, civic behavior, and organizational performance [7,20,25]. Recently, researchers have started giving more attention to its overall impact on organizational progress [20]. It increased the importance of social ties in the working environment [26] ultimately resulting in negative outcomes such as interpersonal deviance, counterproductive behaviors, and performance [8,11]. Moreover, such phenomenon exists in multiple organizational settings [8,27].

\subsection{Job Tension}

Job tension refers to the "psychological reaction of employees to disturbances in objective the perceived work environment " [28] p. 387. Job tension tends to increase the feeling of stress or anxiety, which restricts one's ability to fulfill job demands [29]. According to Lazarus [30], such feeling arises when individuals perceive that they cannot cope with the job demands or with the threats to their well-being. Many factors influence job tension, such as incivility at workplace and dysfunctional behavior of co-workers [31], and leads to an increased level of individual feelings of depression, turnover intention, low level of commitment, perceived injustice and negatively impacts participative behavior [32].

According to Lim and Lee [33], mistreatment at workplace influences an individual's feelings of stress. Such experience of undermining leads to the feeling of injustice and interpersonal aggression, and depression [34]. Employees who feel more job tension become more dissatisfied with their job, perceive less organizational support and are more inclined to leave the organization. The study of Zhu, et al. [6] found that job tension is closely related with the quality of work performance. However, [35] study regarded it as a 'surrogate' which enhances employee defensive behavior and makes them less likely to align with organizational goals. Furthermore, when the climate of workplace deteriorates or becomes uncomfortable, it may be a predictor of a high level of job tension.

\subsection{Employee Loyalty}

Employee loyalty is directly related with the supported and stable workplace. Previous studies defined employee loyalty as an action-oriented concept, which encompasses employee behavior in the extent to which they are committed and responsible for their work or inclined to seek other job opportunities [13,14]. Although the fundamental concept of employee loyalty is emotional attachment, researchers explained it from two perspectives (i) attitudinal (ii) behavioral. In attitudinal perspective, loyalty refers to the feeling of attachment, identification, or employee psychological inclinations towards their organization. However, the behavioral approach is based on employee attitude, which is difficult to measure $[6,32,34,36]$.

Prior research considered employee loyalty as a major driving force to achieve sustainable organizational development $[25,37]$. A loyal workforce can be more committed to organizational goals as they feel that such goals are congruent with their own. Moreover, promoting employee loyalty reduces turnover intention, increases performance, and organizational reputation [14]. Generally, the workforce reaps lucrative benefits in terms of reciprocated loyalty [38], such as high wages. However, employee loyalty improves performance and enhances organizational positive outcomes but when the events do not go well, it may amplify the feeling of stress and tension [39]. 


\section{Hypothesis Development}

According to the COR theory, resources are the capability of individuals to fulfill their basic needs [40,41]. These resources can be from oneself or from the environment (e.g., physical, social, or cognitive), while individuals endeavor to retain and obtain such valued resources to reduce the threat of resource loss [42,43]. Based on the COR theory, insightful perspective explained the influence of workplace ostracism which drains individuals' useful resources required at workplace [36]. In such case, individuals' protection mechanisms may be activated, while to protect further resource loss under such situation, individuals feel continuous stress that can lead to negative outcomes.

Based on the COR theory, we predict that workplace ostracism is a major factor to influence knowledge hiding and increase the feeling of stress. As Connelly, et al. [3] suggested, an increase in alienating behavior can also promote knowledge hiding behavior. Specifically, the likelihood of knowledge hiding behavior increased when employees were unaware of the sanctions and directives regarding the withholding of valuable information [44]. However, the distinctive types of knowledge hiding may have different consequences. Therefore, we examine the following hypothesis:

Hypothesis 1a. Workplace ostracism is positively related with evasive hiding.

Hypothesis $\mathbf{1 b}$. Workplace ostracism is positively related with playing dumb.

Hypothesis 1c. Workplace ostracism is positively related with rationalized hiding.

Workplace ostracism as a work stressor depletes the individual's personal resources, such as self-esteem. Moreover, such "cold shoulder" makes the target feel as an excluded team member, as a result, this limited access to social resources leads to the perception of resource loss [34]. According to the COR theory, to cope with resource loss, an individual may experience psychological strain and to obtain and retain his/her valuable resources, adopt defensive behavior [11]. Workplace ostracism can be the cause of substantial resource loss which impairs individual's capacity to be connected with their work demands, as a result, an individual feels a high level of job tension. Furthermore, to cope with this strain, an individual is more likely to adopt defensive behavior such as knowledge hiding.

Based on the above argument, we predict that workplace ostracism may lead to employees' psychological disturbance and a feeling of job tension. As a consequence, an individual feels stress and psychological strain with depleted resources and such resource loss lowers an individual's ability to meet the job demands and expectations. Hence, this study proposes the following hypothesis:

Hypothesis 2. Workplace ostracism is positively related to job tension.

Employee loyalty describes the reciprocity (emotional or behavioral) between individual and organization [45]. In accordance with the definition of employee loyalty, the attitudinal perspective refers to active job involvement, commitment, and emotional attachment to the organization [46]. Employee loyalty is generally considered as an explicitly positive attribute at an individual and organizational levels. On the contrary, in individuals who experience a challenging or dysfunctional context, their loyalty may be torn between organizational loyalty and their response to that dysfunction such as feeling of threat to their personal wellbeing [47].

Workplace ostracism involves a dysfunction that makes an individual a threat to personal wellbeing. We expect that a positive effect of workplace ostracism exerts on employees a feeling of job tension increased for those who have a high level of employee loyalty toward their organization, because such employees should deplete their personal resources due to the dysfunctional context. Although these employees are committed and want to stay with this organization, social exclusion makes them want to withhold their knowledge. However, organizations apply effective appraisal strategies to retain loyal employees, but these employees may perceive ostracism more hurtful and 
avoid providing access to their knowledge. Moreover, these employees might mobilize their personal resources to cope with workplace ostracism, which may cause loss of resources and arouse feelings of job tension.

In summary, when loyal employees experience workplace ostracism, they tend to perceive more personal and social loss and experience a high level of job tension. Hence, we proposed the following hypotheses

Hypothesis 3. Employee loyalty moderates the relationship between workplace ostracism and job tension such that the relationship is stronger when the employee loyalty is high rather than low.

According to the conservation of resource theory, deleterious effects of stress increase when individuals fail to use coping resources [40] as workplace ostracism as a source of stress involves behavior that is most likely to increase the turnover intention and deviant behaviors [32]. Regarding the mediating role of job tension raised when psychologically stressful circumstances increase at workplace, such stress decreases individuals' capacity to meet job demands and fulfill organization's expectations [28]. As noted earlier, we expect that workplace ostracism exerts more job tension on those who feel more loyalty toward their job, because such employees feel attachment to their job or satisfaction with monetary benefits and want to continue this job. However, these factors can be considered as elementary to enhance ostracism and knowledge hiding association. As such, it is necessary to explore the comprehensive effects of job tension among all types of knowledge hiding (evasive, playing dumb, rationalized).

On the basis of the preceding hypothesis, we further predict the effect of workplace ostracism on knowledge hiding through the mediating role of job tension. Therefore, we also posit that:

Hypothesis 4a. Job tension mediates the relationship between workplace ostracism and evasive hiding.

Hypothesis $\mathbf{4 b}$. Job tension mediates the relationship between workplace ostracism and playing dumb.

Hypothesis 4c. Job tension mediates the relationship between workplace ostracism and rationalized hiding.

\section{Method}

\subsection{Sample and Procedure}

To examine the hypothesis, we collected the data from the textile sector in Pakistan. These organizations are involved in multiple steps of business (production, marketing, and sales). For the purpose of current research, data were collected from middle level employees from different departments, including production, marketing, and sales. However, with the help of HR departments, we obtained a list of 1100 employees, and an e-mail was sent to them, but only 500 employees agreed to participate in this survey. To collect the data, we used a three-wave longitudinal survey with a lag of three months which appears proper to reduce potential common method variance [48] and make it non-vulnerable to dissipate causal effects [16]. Matching codes were used to link the three-step data (i.e., family name, birth date two digits).

In the first step (T1), the participants rated the measures of workplace ostracism, employee loyalty, and demographic information. While in the second step (T2) that was conducted six weeks later, the participants reported their feelings of job tension. After six weeks, in the third step (T3) of data collection, participants rated their knowledge hiding behavior. First, we sent the questionnaire to 500 participants and 488 responded with complete information (response rate $97.6 \%$ ). At T2, participants completed the questionnaire about job tension, the response rate was $84 \%$. Finally, after ignoring incomplete questionnaire at third step we got 392 usable samples, giving a response rate of $95 \%$ of the participant employees, $73 \%$ were male, the mean age was 32.9 years with a standard deviation of 
$5.04,39.1 \%$ reported bachelor degree or above, the mean organizational tenure was 3.96 years with a standard deviation of 2.87 years.

\subsection{Measures}

\subsubsection{Workplace Ostracism}

To measure workplace ostracism, we used 10 item scale [24]. A sample item is "I feel others ignore me at work" using five-point Likert scale (" $1=$ strongly disagree" to " $5=$ strongly agree") while Cronbach's coefficient was 0.945 .

\subsubsection{Knowledge Hiding}

To examine the variable knowledge hiding, we used a scale consisting of 12 items, developed by [3]. Four items were used to measure evasive hiding (e.g., agree to help him/her but never really intended to), and four of them were used to measure playing dumb (e.g., said that I did not know, even though I did), while four items were used to measure rationalized hiding (explained that I would like to tell him/her, but was not supposed to). Respondent completed the measures using five-point Likert scale from $1=$ "strongly disagree" to $5=$ "strongly agree". Cronbach's coefficients were 0.885 , 0.884 , and 0.887 , respectively.

\subsubsection{Job Tension}

To measure job tension, seven items developed by [29] were used. A sample item was "I worked under a great deal of tension". Such scale was used and validated in many studies (e.g., [6,25]). Respondents rated each item using five-point Likert scale ranging from $1=$ "strongly agree" to $5=$ "strongly disagree". Cronbach's alpha for this scale was 0.95 .

\subsubsection{Employee Loyalty}

To measure employee loyalty, eight items were used [49]. For example, "I would like to spend the rest of my career in this organization". Respondents rated each item from $1=$ "strongly agree" to $5=$ "strongly disagree", while Cronbach's alpha for the scale was 0.91 .

\section{Results}

In latent variable modeling, structural equation modeling (SEM) is a useful tool. There are two types of frequently applied methods, named covariance-based (CB-SEM) and variance-based (PLS-SEM). Within SEM modeling, both methods are widely applied. However, CB-SEM focuses on minimizing the distance between the observed and predicted covariance matrices, while PLS-SEM focuses on maximizing the explained variances of the latent variables. Comparing them, it can be observed that CB-SEM works under stricter conditions which can be summarized as follows: In the aspect of the assumption of application, CB-SEM works under the assumption of normality, while PLS-SEM can work without normality assumption. For CB-SEM, the sample size should be large, while PLS-SEM can work with a relatively small sample size. The main focus of research using CB-SEM is the theory testing, whereas the focus for using PLS-SEM is parameter testing. For examination of fit mode, there are several indices for measuring global-fit in using CB-SEM, while in the case of PLS-SEM, testing of path coefficients can be implemented with the help of bootstrapping algorithm (one can see details in [50]). Therefore, this study used the partial least square (PLS) technique for data analysis by using SmartPLS 3.0 software to examine the research model. The main advantage of using PLS methods is that they accommodate the latent constructs to be modeled either as reflective or formative constructs and have a lower restriction regarding sample size [51-53]. A listwise deletion of missing values reduced our sample from 448 to 392 . The hierarchical component model and a two-stage approach were used [54]. The item loading, reliability, validity, and assessment measurement model were examined in the first stage, while estimating the path coefficients of the structural model was 
established in the second stage. The correlation between variables along with means and standard deviations are presented in Table 1. All correlations are significant at $\alpha=0.001$.

Table 1. Descriptive and correlation matrix.

\begin{tabular}{|c|c|c|c|c|c|c|c|c|}
\hline & $\mathbf{M}$ & SD & (1) & (2) & (3) & (4) & (5) & (6) \\
\hline Workplace Ostracism (1) & 3.434 & 0.922 & 1.000 & & & & & \\
\hline Job Tension (2) & 3.183 & 1.035 & $0.515^{* *}$ & 1.000 & & & & \\
\hline Employee Loyalty (3) & 3.194 & 0.881 & $0.255^{* *}$ & $0.557 * *$ & 1.000 & & & \\
\hline Evasive Hiding (4) & 3.344 & 1.021 & $0.447^{* *}$ & $0.558^{* *}$ & $0.460 * *$ & 1.000 & & \\
\hline Playing Dumb (5) & 3.308 & 0.990 & $0.429 * *$ & $0.572 * *$ & $0.441^{* *}$ & $0.254 * *$ & 1.000 & \\
\hline Rationalized Hiding (6) & 3.025 & 0.936 & $0.125 *$ & -0.012 & -0.038 & 0.027 & -0.014 & 1.000 \\
\hline
\end{tabular}

\subsection{Measurement Model Evaluation}

The aim of measurement model evaluation is to assess the consistency and validity of the variables. Consistency is evaluated through induvial variables and construct reliability tests, while validity evaluations are through convergent and discriminant validity tests [55]. All the first-order variables were measured reflectively. Table 2 represents the results of each measurement scale (average variance extracted, composite reliability, and Cronbach's alpha) along with the values of convergent validity measures. All values of composite reliability and Cronbach's alpha are greater than 0.70 , indicating high levels of internal consistency reliability [56]. The next measure is convergent validity, which is defined as the model's ability to explain the indicator's variance. Fornell et al. [57] suggested average variance extracted (AVE) can provide evidence for convergent validity and [55] suggested the threshold for AVE to be 0.5. In this study, the AVE ranges from 0.608 to 0.755 well above the required minimum level of 0.50 [55], which represents the high level of convergent validity. Finally, the discriminant validity of this model assessed by [57], defined as the square root of average variance extracted (AVE) of each latent variable, should be greater than the correlation. Table 2 clearly shows that the variables fulfill Fornell-Larcker criterion and discriminant validity is met for this research. The confirmatory factor analysis (outer loadings) and path coefficients are presented graphically in Figure 1. All reflective loadings range from 0.767 to 0.926 and are significant at the 0.001 level.

Table 2. Discriminant validity (Fornell-Larcker criterion).

\begin{tabular}{cccccccccc}
\hline & AVE & CR & Cronbach's Alpha & (1) & (2) & (3) & (4) & (5) & (6) \\
\hline Workplace ostracism (1) & 0.670 & 0.953 & 0.945 & $\mathbf{0 . 8 1 9}$ & & & & & \\
Job tension (2) & 0.755 & 0.956 & 0.946 & 0.519 & $\mathbf{0 . 8 6 9}$ & & & & \\
Employee loyalty (3) & 0.608 & 0.926 & 0.908 & 0.227 & 0.559 & $\mathbf{0 . 7 8 0}$ & & & \\
Evasive hiding (4) & 0.743 & 0.920 & 0.885 & 0.448 & 0.562 & 0.464 & $\mathbf{0 . 8 6 2}$ & & \\
Playing dumb (5) & 0.743 & 0.920 & 0.884 & 0.428 & 0.576 & 0.443 & 0.256 & $\mathbf{0 . 8 6 2}$ & \\
Rationalized hiding (6) & 0.735 & 0.917 & 0.883 & 0.129 & -0.019 & -0.044 & 0.019 & -0.129 & $\mathbf{0 . 8 1 9}$ \\
\hline
\end{tabular}

\subsection{Structural Model Evaluation}

The structural model assesses the relationship between exogenous and endogenous latent variables through the coefficient of determination $R^{2}$ [58] and path coefficients of the model, that is, $\beta$ values [59]. $R^{2}$ represents the degree of explained variance of endogenous latent variable [60] while $\beta$ indicates the strength of an effect from variables to endogenous latent variables [61].

According to [62,63], for a good model, the value of $R^{2}$ of the endogenous latent variable should be more than 0.26 . Here in our model, the value of $R^{2}$ for rationalized hiding is very low, but for all other variables is higher than the suggested value, the model is considered to have a substantial degree of explained variance of knowledge hiding by factors considered in this study. We also calculated the effect sizes $f^{2}$ and $q^{2}$ for knowledge hiding. The values of $f^{2}$ and $q^{2}$ are $(0.2258,0.2632,0.0103)$ and $(0.1327,0.1576,0.0051)$, respectively, for knowledge hiding (evasive hiding, playing dumb, and 
rationalized hiding). The next step is assessing the path coefficient of all latent variables (paths) by comparing $\beta$ values among all the paths. The highest $\beta$ value symbolizes the strongest effect of predictor (exogenous) latent variable towards the dependent (endogenous) latent variable [64].

The test is achieved by performing the nonparametric bootstrapping technique $[59,65,66]$. The bootstrapping technique computes $t$-value by creating a pre-specified number of samples.

In this study, bootstrapping generated 5000 samples and these samples are used to compute $t$-values, as presented in Tables 3 and 4 . Table 3 represents the PLS algorithm and bootstrapping results of the model with and without moderation effect and Table 4 represents the results of the mediation analysis. From Table 3, the relationship between workplace ostracism and knowledge hiding is highly significant $(\beta=0.2142, p<0.001),(\beta=0.1775, p<0.001)$ and $(\beta=0.1899, p<0.001)$ for evasive hiding, playing dumb, and rationalized hiding, respectively, supporting $\mathrm{H}_{1}\left(\mathrm{H}_{1 \mathrm{a}}, \mathrm{H}_{1 \mathrm{~b}}, \mathrm{H}_{1 \mathrm{c}}\right)$. Similarly, $\mathrm{H}_{2}$ is also supported by the highly significant relation between workplace ostracism and job tension in both models (with and without moderator effect). The path coefficient values of workplace ostracism and job tension for the model without and with moderation effect are $(\beta=0.3134, p<0.001)$ and $(\beta=0.3888, p<0.001)$, respectively. The value of $R^{2}$ for job tension without moderation effect is 0.475 , while the $R^{2}$ value for job tension with moderation effect is 0.500 . Both the beta coefficient and $R^{2}$ increased by adding employee loyalty as moderator in addition, the moderation effect of job tension is also positive and significant as $(\beta=0.1794, p<0.001)$ supporting $\mathrm{H}_{3}$. We also presented a moderated relationship in Figure 2, which indicates that employee loyalty strengthens the positive relationship between workplace ostracism and job tension.

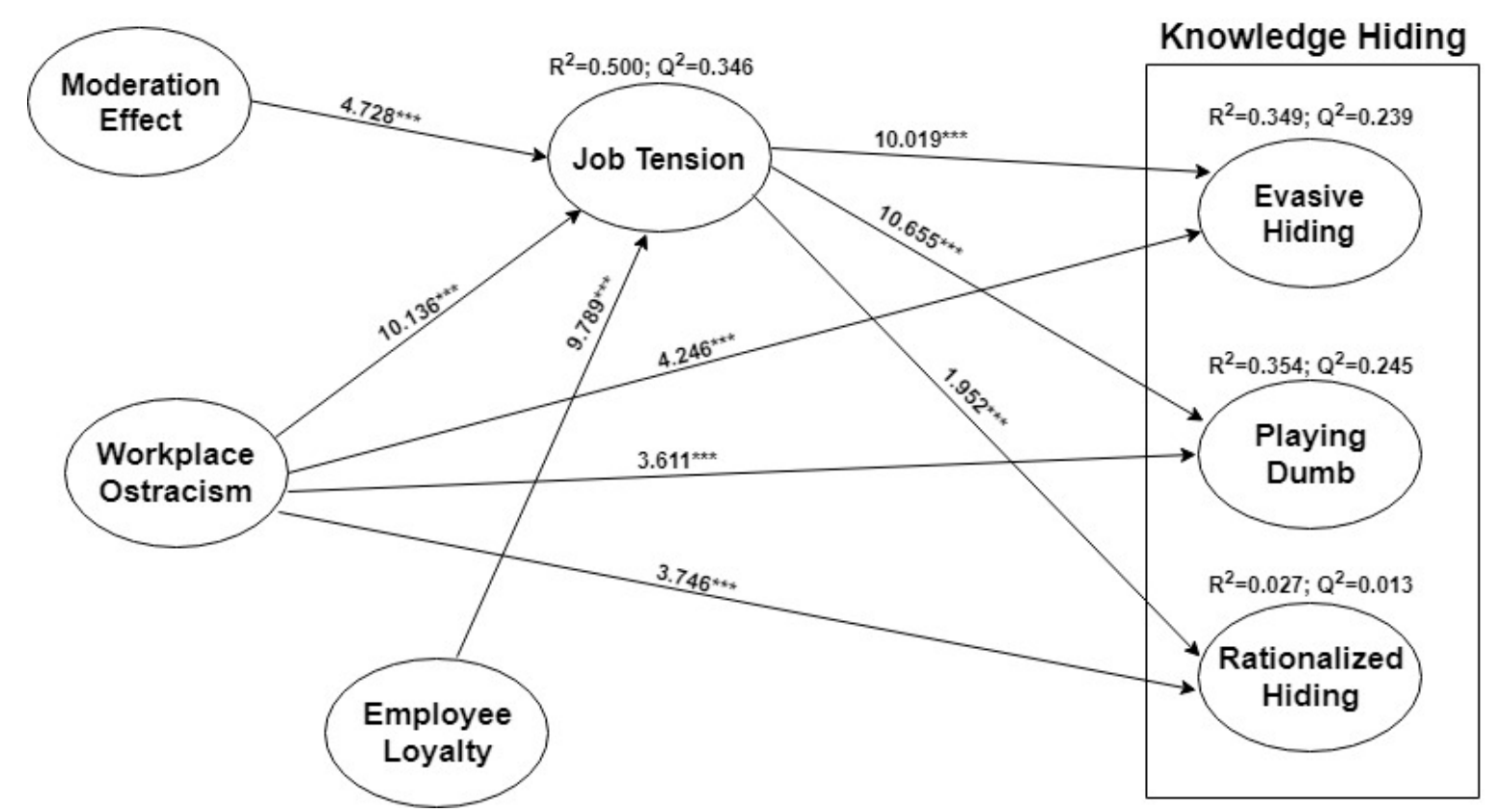

Figure 2. Assessment of structural model. (Note: ${ }^{* * *}$ represents the significant at $0.1 \%$ ). 
Table 3. Significant testing results of the structural model path coefficients.

\begin{tabular}{ccccccc}
\hline $\begin{array}{c}\text { Without Moderation } \\
\text { Effect Path }\end{array}$ & Path Coefficients & Mean & SD & $t$ Values & $p$ Values & CI (2.5-97.5)\% \\
\hline EL $\rightarrow$ JT & 0.4654 & 0.4665 & 0.0372 & 12.5109 & 0.0000 & $(0.3902,0.5360)$ \\
WO $\rightarrow$ EH & 0.2142 & 0.2156 & 0.0519 & 4.1303 & 0.0000 & $(0.1110,0.3152)$ \\
WO $\rightarrow$ JT & 0.4134 & 0.4140 & 0.0382 & 10.8223 & 0.0000 & $(0.3359,0.4869)$ \\
WO $\rightarrow$ PD & 0.1775 & 0.1782 & 0.0488 & 3.6396 & 0.0003 & $(0.0809,0.2760)$ \\
WO $\rightarrow$ RH & 0.1899 & 0.1948 & 0.0519 & 3.6589 & 0.0003 & $(0.0953,0.2967)$ \\
\hline With Moderation & Path Coefficients & Mean & SD & $t$ Values & $p$ Values & CI (2.5-97.5)\% \\
Effect Path & & & & & \\
\hline EL $\rightarrow$ JT & 0.4201 & 0.4184 & 0.0422 & 9.9635 & 0.0000 & $(0.3320,0.4992)$ \\
WO $\rightarrow$ EH & 0.2142 & 0.2157 & 0.0508 & 4.2138 & 0.0000 & $(0.1146,0.3136)$ \\
WO $\rightarrow$ JT & 0.3888 & 0.3859 & 0.0384 & 10.1222 & 0.0000 & $(0.3189,0.4600)$ \\
WO $\rightarrow$ PD & 0.1775 & 0.1781 & 0.0488 & 3.6394 & 0.0003 & $(0.0807,0.2714)$ \\
WO $\rightarrow$ RH & 0.1899 & 0.1961 & 0.0515 & 3.6886 & 0.0002 & $(0.0960,0.2949)$ \\
Moderation Effect $\rightarrow$ JT & 0.1794 & 0.1899 & 0.0376 & 4.7767 & 0.0000 & $(0.1170,0.2642)$ \\
\hline
\end{tabular}

Table 4. Summary of Mediation effects test.

\begin{tabular}{|c|c|c|c|c|c|c|c|}
\hline Direct Effects Path & Path Coefficients & Mean & SD & $t$ Values & $p$ Values & $\begin{array}{c}\mathrm{CI} \\
(2.5-97.5) \%\end{array}$ & $\begin{array}{c}\text { BC } \\
(2.5-97.5) \%\end{array}$ \\
\hline $\mathrm{JT} \rightarrow \mathrm{EH}\left(b_{1}\right)$ & 0.4506 & 0.4506 & 0.0450 & 10.0185 & 0.0000 & $\begin{array}{l}(0.3608, \\
0.5363)\end{array}$ & $\begin{array}{l}(0.3608, \\
0.5363)\end{array}$ \\
\hline $\mathrm{JT} \rightarrow \mathrm{PD}\left(b_{2}\right)$ & 0.4834 & 0.4840 & 0.0454 & 10.6548 & 0.0000 & $\begin{array}{l}(0.3931, \\
0.5723)\end{array}$ & $\begin{array}{l}(0.3931, \\
0.5723)\end{array}$ \\
\hline $\mathrm{JT} \rightarrow \mathrm{RH}\left(b_{3}\right)$ & -0.1179 & -0.1208 & 0.0604 & 1.9517 & 0.0510 & $\begin{array}{c}(-0.2337, \\
0.0032)\end{array}$ & $\begin{array}{c}(-0.2337 \\
0.0032)\end{array}$ \\
\hline $\mathrm{WO} \rightarrow \mathrm{EH}\left(c_{1}^{\prime}\right)$ & 0.2142 & 0.2154 & 0.0505 & 4.2458 & 0.0000 & $\begin{array}{l}(0.1164 \\
0.3139)\end{array}$ & $\begin{array}{l}(0.1164, \\
0.3139)\end{array}$ \\
\hline $\mathrm{WO} \rightarrow \mathrm{JT}(\mathrm{a})$ & 0.3888 & 0.3873 & 0.0384 & 10.1363 & 0.0000 & $\begin{array}{l}(0.3105, \\
0.4611)\end{array}$ & $\begin{array}{l}(0.3105 \\
0.4611)\end{array}$ \\
\hline
\end{tabular}

Table 4. Cont.

\begin{tabular}{|c|c|c|c|c|c|c|c|}
\hline Direct Effects Path & Path Coefficients & Mean & SD & $t$ Values & $p$ Values & $\begin{array}{c}\text { CI } \\
(2.5-97.5) \%\end{array}$ & $\begin{array}{c}\text { BC } \\
(2.5-97.5) \%\end{array}$ \\
\hline $\mathrm{WO} \rightarrow \mathrm{PD}\left(c_{2}^{\prime}\right)$ & 0.1775 & 0.1781 & 0.0491 & 3.6106 & 0.0003 & $\begin{array}{l}(0.0789, \\
0.2731)\end{array}$ & $\begin{array}{l}0.0789, \\
0.2731)\end{array}$ \\
\hline $\mathrm{WO} \rightarrow \mathrm{RH}\left(c_{3}^{\prime}\right)$ & 0.1900 & 0.1953 & 0.0507 & 3.7462 & 0.0002 & $\begin{array}{l}(0.0986, \\
0.2939)\end{array}$ & $\begin{array}{l}(0.0986, \\
0.2939)\end{array}$ \\
\hline Indirect Effects & Path Coefficients & Mean & SD & $p$ Values & $\begin{array}{c}\mathrm{CI} \\
(2.5-97.5) \%\end{array}$ & $\begin{array}{c}\mathrm{BC} \\
(2.5-97.5) \%\end{array}$ & VAF \\
\hline$H_{4 a}: a \times b_{1}$ & 0.1754 & 0.1745 & 0.0249 & 0.0000 & $\begin{array}{l}(0.1284, \\
0.2249)\end{array}$ & $\begin{array}{l}0.1312, \\
02284)\end{array}$ & $45.05 \%$ \\
\hline$H_{4 b}: a \times b_{2}$ & 0.1879 & 0.1875 & 0.0260 & 0.0000 & $\begin{array}{l}(0.1390, \\
0.2407)\end{array}$ & $\begin{array}{l}(0.1413, \\
0.2451)\end{array}$ & $51.49 \%$ \\
\hline$H_{4 c}: a \times b_{c}$ & -0.0459 & -0.0468 & 0.0241 & 0.0572 & $\begin{array}{c}(-0.0931 \\
0.0015)\end{array}$ & $\begin{array}{c}(-0.0907, \\
0.0049)\end{array}$ & $19.46 \%$ \\
\hline
\end{tabular}

CI: Confidence interval. BC: Bias corrected. VAF: Variance accounted for.

\subsection{Mediation Assessment}

This study analyzes the significance of three mediation effects by adopting the bootstrapping method $[67,68]$. The requirement for the mediation is that the indirect effect has to be significant and $95 \%$ bootstrapped confidence interval should not contain the value zero. For the testing of our hypothesis 4 , that is, job tension will mediate the relationship of workplace ostracism and knowledge hiding (evasive hiding, playing dumb, and rationalized hiding), we checked both direct and indirect effects. First, we checked the significance of indirect effect by running 5000 bootstrapping samples and 
found indirect effects on hypotheses $\mathrm{H}_{4 \mathrm{a}}, \mathrm{H}_{4 \mathrm{~b}}$, and $\mathrm{H}_{4 \mathrm{c}}$ with coefficient values $(\beta=0.1753, p<0.001)$, $(\beta=0.1880, p<0.001)$, and $(\beta=-0.0456, p>0.001)$, respectively. The results are presented in Table 4. Here, the indirect effect of workplace ostracism and rationalized hiding is insignificant, which indicates no mediation. We used total effects and variance account for (VAF) to examine the strength of mediation by following Nitzl et al. [69]. The VAF values for the indirect effects of workplace ostracism with evasive hiding, playing dumb, and rationalized hiding are $0.4505,0.5149$, and 0.1946 , respectively. We can conclude that job tension partially mediates the relationship between workplace ostracism and evasive hiding as well as playing dumb (for $20 \%<\mathrm{VAF}<80 \%$ ). As VAF $<20 \%$ for $\mathrm{H}_{4 c}$, so, we can conclude that there is no mediation effect of job tension between workplace ostracism and rationalized hiding.

\section{Discussion}

The present study investigated the effect of workplace ostracism on individuals' knowledge hiding behavior. Four hypotheses were articulated to test the model. The first hypothesis is related to the positive relationship among workplace ostracism and evasive hiding, playing dumb, whereas not related with rationalized hiding. As claimed, a significant positive association was found between workplace ostracism and job tension, which implies that workplace ostracism depletes resources and targets feel psychological stress and disturbance to fulfill job demands. These findings are consistent with $[6,20]$. In the third hypothesis we posited that employee loyalty moderates the relationship of workplace ostracism and job tension, results indicate that a high level of employee loyalty increases job tension. The rationale for such positive association could be the fact that employees want to stay with this job but social exclusion reaps the benefits of such loyalty and increases psychological stress, which restricts them in fulfilling their job demands. Hence, it can be asserted that organizations not only focus on pay incentives but also provide a justified and desirable attitudinal environment to increase the benefits of employee loyalty. Furthermore, the relationship among workplace ostracism and knowledge hiding (evasive hiding and play dump) is significantly mediated through job tension. These results indicate that job tension as psychological stress plays a vital role in increasing the knowledge hiding behavior consistent with results of Zhao et al. [4]. Based on the results, high level of loyalty prevents employees from withdrawing from the job but such workplace ostracism enhances job tension and influences individuals' behavior negatively as they become more likely to adopt knowledge hiding behavior.

\subsection{Theoretical Implications}

The present study advances the existing literature in many ways. First, as knowledge is an "intellectual asset" and organization cannot coerce workers to transfer, the individual's willingness is the main imperative. Therefore, it is critical to explore the individual antecedents that promote knowledge hiding behavior. The seminal work of $[11,70]$ and prior research explained the knowledge hiding behavior and predicted many other influential variables, such as interpersonal antecedents (peers, supervisor relationship), social cognitive (culture, justice), individual abilities (i.e., emotional intelligence, political skills) $[1,8,12]$. In line with this reasoning, the current study seeks to extend the growing literature by identifying that mistreatment at work, such as workplace ostracism, promotes knowledge hiding behavior as this kind of mistreatment at workplace negatively affects the attitude, behavior and performance of the target [5]. Considering this, we enrich the literature, by showing that workplace ostracism works as a stressor. As we hypothesized, individuals cope their threatened resources through engaging in knowledge hiding behavior consistent with results of $[1,25,70,71]$.

Secondly, in addition to exploring the main relationship among workplace ostracism and knowledge hiding, the current study also anticipated job tension as a mediator. Based on such association, the present research is a response to the call by previous studies (i.e., $[1,11,70])$, to identify the variables that divert individual behavior towards knowledge hiding. Moreover, this study also contributed by providing a better understanding of individual cognition effect from the perspective 
of the COR theory, which promotes un-participative behavior like knowledge hiding. As predicted, an increase in job stress raises emotional disturbance and strengthens behavioral response, such as knowledge hiding behavior. These findings not only contribute to the knowledge hiding literature by going beyond the scope of the underlying mechanisms of knowledge hiding, but also address the call for integrating knowledge hiding behavior and mistreatment at work theoretical frameworks $[11,25,70]$.

The third contribution to the knowledge is the establishment of a link between workplace ostracism and job tension by testing the moderating effects of employee loyalty. However, our results support the argument that workplace ostracism exhibits worse results on those employees who have a high level of employee loyalty. As [14] noted, low level of employee loyalty inclined individuals' more towards withdrawal but a high level of loyalty increased the negative behavioral response. Moreover, the present study addressed the call for greater identification of moderators which may enhance the negative effects of workplace ostracism (e.g., $[7,8]$ ). As such, these findings contribute to the literature through employing an individual's cognition and feeling of stress effects on behavior in a single model. Furthermore, the research emphasized the importance of social justice at workplace, although monetary benefits can increase the intention to stay with such organization, workplace environment is the key to get maximum performance.

\subsection{Managerial Implications}

Although previous research $[1,70]$ considered knowledge hiding more risky for organizational success, empirical research is relatively limited, which makes the present research more salient. The current study indicates that, workplace ostracism acts as a key factor to influence the knowledge hiding behavior at work. According to the results, ostracized individuals feel more job tension and depleted personal resources, which activates the resource retaining mechanism and influences knowledge hiding behavior. Therefore, workplace ostracism is costly for any organization and needs some preventive measures, such as cultivating the culture which promotes interpersonal interaction, and team building, to decrease its negative effects. Furthermore, encouraging the cooperative goal interdependence can be a helpful measure to decrease workplace ostracism [4], task interdependence and team-based reward system foster the cooperative environment and prevent workplace ostracism [10].

Furthermore, vertical business organizations need to pay more cost than any other organization if their employees feel job tension [28]. As noted, workplace ostracism depletes the employee resources and they feel job tension which drains their resources. To detect such issue, organizations need to adopt early intervention strategies, such as an effective communication system and coaching to overcome employee stress. Moreover, managers should also observe and try to find the cause of employee stress, provide them with professional psychological guidance and encourage employee participation. However, 360 pieces of feedback can also be a useful tool to evaluate the individual's feeling about others' behavior.

In addition, job tension impedes employee participating behavior, and they become more inclined to hide knowledge. As research has shown, knowledge hiding is not the opposite of knowledge sharing [3], it is not enough to promote a knowledge sharing environment, but attenuating knowledge hiding behavior is also as important. Furthermore, to overcome the reasons for knowledge hiding behavior, organizations need to foster a culture of task interdependence and teamwork [6].

Finally, this study explained the effects of employees' positive attitudes towards their organization, while monetary incentives are not enough to reduce negative outcomes. Therefore, mangers need to pay more attention towards moral motivation to improve job tension management. The present research findings show that it becomes a key challenge to achieve organizational goals, but reducing workplace ostracism, ensuring a collaborative environment and enhancing well-being can benefit the organization. 


\subsection{Limitations and Future Research}

However, this study contributes to the existing body of literature in many ways, but can also be interpreted in light of certain limitations. First, the generalizability of the results may be contaminated because data were collected from one industry, while the effects may vary with job demands, culture, and job mobility. As such, testing this model in different organizational settings and cultural contexts may provide some other interesting findings. Secondly, although the time-lagged design has been used to establish the cause and effect relationship among perceived ostracism and knowledge hiding behavior, such relation may not be fully explained until the data correlational in nature. In addition, some other individual abilities and differences, such as personality types and family conflicts, may also lead to more stress (Connelly and Zweig, 2015) [15]. Furthermore, it is possible that regarding job tenure, most likely employees with less tenure perceive more ostracism, while employees with high tenure are more likely to hide knowledge [6,72]. Hence, for future research we recommend a longitudinal design with more precise demographic information.

\section{Conclusions}

This research developed a moderating mediated model to examine the reasons behind knowledge hiding behavior. Findings show that workplace ostracism as a major work stressor promotes such behavior at work. Moreover, we examined the boundary conditions under which individuals are more likely to withhold requested knowledge. As predicted, findings show that workplace ostracism positively affects the individual' feeling of job tension while such relation becomes stronger for individuals who have a high level of employee loyalty. In addition, organizations could suffer more from the negative effects of workplace ostracism as employees have a high level of job tension. Furthermore, this study also provides several paths for mangers, as well as researchers, to devote more attention to knowledge hiding behavior related with individuals' attitudes towards their organization.

Author Contributions: S.R. and Y.X. formulated the study design, conceived and designed the research methodology. S.R. and S.H. collected and analyzed the data; S.R. wrote the paper with the help of Y.X.

Funding: This research was funded by Xu Yusen grant number G0203, 71272095.

Acknowledgments: This research work was supported by the National Natural Science Foundation of China under Grant No. G0203, 71272095. The useful comments and suggestions from the editor and all the reviewers are extremely appreciated.

Conflicts of Interest: The authors declare no conflict of interest.

\section{References}

1. Yang, J.-T. Knowledge sharing: Investigating appropriate leadership roles and collaborative culture. Tour. Manag. 2007, 28, 530-543. [CrossRef]

2. Bartol, K.M.; Srivastava, A. Encouraging knowledge sharing: The role of organizational reward systems. J. Leadersh. Organ. Stud. 2002, 9, 64-76. [CrossRef]

3. Connelly, C.E.; Zweig, D.; Webster, J.; Trougakos, J.P. Knowledge hiding in organizations. J. Organ. Behav. 2012, 33, 64-88. [CrossRef]

4. Zhao, H.; Xia, Q.; He, P.; Sheard, G.; Wan, P. Workplace ostracism and knowledge hiding in service organizations. Int. J. Hosp. Manag. 2016, 59, 84-94. [CrossRef]

5. O'Reilly, J.; Robinson, S.L.; Berdahl, J.L.; Banki, S. Is negative attention better than no attention? The comparative effects of ostracism and harassment at work. Organ. Sci. 2014, 26, 774-793. [CrossRef]

6. Zhu, H.; Lyu, Y.; Deng, X.; Ye, Y. Workplace ostracism and proactive customer service performance: A conservation of resources perspective. Int. J. Hosp. Manag. 2017, 64, 62-72. [CrossRef]

7. Balliet, D.; Ferris, D.L. Ostracism and prosocial behavior: A social dilemma perspective. Organ. Behav. Hum. Decis. Processes 2013, 120, 298-308. [CrossRef]

8. Ferris, D.L.; Lian, H.; Brown, D.J.; Morrison, R. Ostracism, self-esteem, and job performance: When do we self-verify and when do we self-enhance? Acad. Manag. J. 2015, 58, 279-297. [CrossRef] 
9. Chung, Y.W.; Yang, J.Y. The mediating effects of organization-based self-esteem for the relationship between workplace ostracism and workplace behaviors. Balt. J. Manag. 2017, 12, 255-270. [CrossRef]

10. Wu, C.-H.; Liu, J.; Kwan, H.K.; Lee, C. Why and when workplace ostracism inhibits organizational citizenship behaviors: An organizational identification perspective. J. Appl. Psychol. 2016, 101, 362. [CrossRef]

11. Zhao, H.; Peng, Z.; Sheard, G. Workplace ostracism and hospitality employees' counterproductive work behaviors: The joint moderating effects of proactive personality and political skill. Int. J. Hosp. Manag. 2013, 33, 219-227. [CrossRef]

12. Andersson, L.M.; Pearson, C.M. Tit for tat? The spiraling effect of incivility in the workplace. Acad. Manag. Rev. 1999, 24, 452-471. [CrossRef]

13. Eskildsen, J.K.; Nussler, M.L. The managerial drivers of employee satisfaction and loyalty. Total Qual. Manag. 2000, 11, 581-588. [CrossRef]

14. Guillon, O.; Cezanne, C. Employee loyalty and organizational performance: A critical survey. J. Organ. Chang. Manag. 2014, 27, 839-850. [CrossRef]

15. Connelly, C.E.; Zweig, D. How perpetrators and targets construe knowledge hiding in organizations. Eur. J. Work Organ. Psychol. 2015, 24, 479-489. [CrossRef]

16. Peng, H. Why and when do people hide knowledge? J. Knowl. Manag. 2013, 17, 398-415. [CrossRef]

17. Al Qudah, N.F.; Yang, Y.; Anjum, M.A. Transformational Training Programs and Quality Orientation of Employees: Does Employees' Loyalty Matter? Sustainability 2018, 10, 465. [CrossRef]

18. Andreeva, T.; Kianto, A. Does knowledge management really matter? Linking knowledge management practices, competitiveness and economic performance. J. Knowl. Manag. 2012, 16, 617-636. [CrossRef]

19. Lin, H.-F. Linking knowledge management orientation to balanced scorecard outcomes. J. Knowl. Manag. 2015, 19, 1224-1249. [CrossRef]

20. Zhao, H.; Xia, Q. An examination of the curvilinear relationship between workplace ostracism and knowledge hoarding. Manag. Decis. 2017, 55, 331-346. [CrossRef]

21. Von der Trenck, A. "It's Mine." The Role of Psychological Ownership and Territoriality in Knowledge Hiding. Proceedings of ICIS Proceedings; Available online: https://www.semanticscholar. org/paper/\%22It|T1 \textquoterights-Mine.\%22-The-Role-of-Psychological-Ownership-in-Trenck/ b5ea4f3b13903ad6991b3dc17d83507bc5861965 (accessed on 8 October 2019).

22. Webster, J.; Brown, G.; Zweig, D.; Connelly, C.E.; Brodt, S.; Sitkin, S. Beyond knowledge sharing: Withholding knowledge at work. In Research in Personnel and Human Resources Management; Emerald Group Publishing Limited: Bingley, UK, 2008; pp. 1-37.

23. Chung, Y.W. Workplace ostracism and workplace behaviors: A moderated mediation model of perceived stress and psychological empowerment. Anxiety Stress Coping 2018, 31, 304-317. [CrossRef] [PubMed]

24. Ferris, D.L.; Brown, D.J.; Berry, J.W.; Lian, H. The development and validation of the Workplace Ostracism Scale. J. Appl. Psychol. 2008, 93, 1348-1366. [CrossRef] [PubMed]

25. Wu, L.Z.; Yim, F.H.k.; Kwan, H.K.; Zhang, X. Coping with workplace ostracism: The roles of ingratiation and political skill in employee psychological distress. J. Manag. Stud. 2012, 49, 178-199. [CrossRef]

26. Wesselmann, E.D.; Wirth, J.H.; Pryor, J.B.; Reeder, G.D.; Williams, K.D. When do we ostracize? Soc. Psychol. Personal. Sci. 2013, 4, 108-115. [CrossRef]

27. Hales, A.H.; Kassner, M.P.; Williams, K.D.; Graziano, W.G. Disagreeableness as a cause and consequence of ostracism. Personal. Soc. Psychol. Bull. 2016, 42, 782-797. [CrossRef]

28. Chisholm, R.F.; Kasl, S.V.; Eskenazi, B. The nature and predictors of job related tension in a crisis situation: Reactions of nuclear workers to the Three Mile Island accident. Acad. Manag. J. 1983, 26, 385-405.

29. House, R.J.; Rizzo, J.R. Toward the measurement of organizational practices: Scale development and validation. J. Appl. Psychol. 1972, 56, 388. [CrossRef]

30. Lazarus, R.S. Psychological Stress and the Coping Process; Mc Graw-Hill: New York, NY, USA, 1966.

31. Johnson, P.R.; Indvik, J. Rudeness at work: Impulse over restraint. Public Pers. Manag. 2001, 30, 457-465. [CrossRef]

32. Robinson, S.L.; Wang, W.; Kiewitz, C. Coworkers behaving badly: The impact of coworker deviant behavior upon individual employees. Annu. Rev. Organ. Psychol. Organ. Behav. 2014, 1, 123-143. [CrossRef]

33. Lim, S.; Lee, A. Work and nonwork outcomes of workplace incivility: Does family support help? J. Occup. Health Psychol. 2011, 16, 95. [CrossRef] 
34. Duffy, M.K.; Ganster, D.C.; Shaw, J.D.; Johnson, J.L.; Pagon, M. The social context of undermining behavior at work. Organ. Behav. Hum. Decis. Process. 2006, 101, 105-126. [CrossRef]

35. Lyu, Y.; Zhu, H.; Zhong, H.-J.; Hu, L. Abusive supervision and customer-oriented organizational citizenship behavior: The roles of hostile attribution bias and work engagement. Int. J. Hosp. Manag. 2016, 53, 69-80. [CrossRef]

36. Leung, A.S.; Wu, L.; Chen, Y.; Young, M.N. The impact of workplace ostracism in service organizations. Int. J. Hosp. Manag. 2011, 30, 836-844. [CrossRef]

37. Williams, K.D. Ostracism: A temporal need-threat model. Adv. Exp. Soc. Psychol. 2009, 41, 275-314.

38. Zatzick, C.D.; Deery, S.J.; Iverson, R.D. Understanding the determinants of who gets laid off: Does affective organizational commitment matter? Hum. Resour. Manag. 2015, 54, 877-891. [CrossRef]

39. Kraemer, T.; HJ Gouthier, M. How organizational pride and emotional exhaustion explain turnover intentions in call centers: A multi-group analysis with gender and organizational tenure. J. Serv. Manag. 2014, 25, 125-148. [CrossRef]

40. Hobfoll, S.E. The influence of culture, community, and the nested-self in the stress process: Advancing conservation of resources theory. Appl. Psychol. 2001, 50, 337-421. [CrossRef]

41. Wang, M.; Liao, H.; Zhan, Y.; Shi, J. Daily customer mistreatment and employee sabotage against customers: Examining emotion and resource perspectives. Acad. Manag. J. 2011, 54, 312-334. [CrossRef]

42. Hobfoll, S.E. Conservation of resources: A new attempt at conceptualizing stress. Am. Psychol. 1989, 44, 513. [CrossRef]

43. Hobfoll, S.E. Social and psychological resources and adaptation. Rev. Gen. Psychol. 2002, 6, 307. [CrossRef]

44. Gkorezis, P.; Bellou, V. The relationship between workplace ostracism and information exchange: The mediating role of self-serving behavior. Manag. Decis. 2016, 54, 700-713. [CrossRef]

45. Meyer, J.P.; Becker, T.E.; Vandenberghe, C. Employee commitment and motivation: A conceptual analysis and integrative model. J. Appl. Psychol. 2004, 89, 991. [CrossRef] [PubMed]

46. Rajput, S.; Singhal, M.; Tiwari, S. Job satisfaction and employee loyalty: A study of academicians. Asian J. Manag. 2016, 7, 105-109. [CrossRef]

47. Johnson, L.U.; Rogers, A.; Stewart, R.; David, E.M.; Witt, L. Effects of politics, emotional stability, and LMX on job dedication. J. Leadersh. Organ. Stud. 2017, 24, 121-130. [CrossRef]

48. Podsakoff, N. Common method biases in behavioral research: A critical review of the literature and recommended remedies. J. Appl. Psychol. 2003, 88, 879-903. [CrossRef] [PubMed]

49. Allen, N.J.; Meyer, J.P. The measurement and antecedents of affective, continuance and normative commitment to the organization. J. Occup. Organ. Psychol. 1990, 63, 1-18. [CrossRef]

50. Kazár, K. PLS Path Analysis and its Application for the Examination of the Psychological Sense of a Brand Community. Procedia Econ. Financ. 2014, 17, 183-191. [CrossRef]

51. Chin, W.W.; Marcolin, B.L.; Newsted, P.R. A partial least squares latent variable modeling approach for measuring interaction effects: Results from a Monte Carlo simulation study and an electronic-mail emotion/adoption study. Inf. Syst. Res. 2003, 14, 189-217. [CrossRef]

52. Lee, C.-S.; Chen, Y.-C.; Tsui, P.-L.; Yu, T.-H. Examining the relations between open innovation climate and job satisfaction with a PLS path model. Qual. Quant. 2014, 48, 1705-1722. [CrossRef]

53. Terzi, S.; Trezzini, A.; Moroni, L. A PLS path model to investigate the relations between institutions and human development. Qual. Quant. 2014, 48, 1271-1290. [CrossRef]

54. Ringle, C.M.; Sarstedt, M.; Straub, D. A critical look at the use of PLS-SEM in MIS Quarterly. MIS Q. 2012, 36, iii-xiv. [CrossRef]

55. Bagozzi, R.P.; Yi, Y. On the evaluation of structural equation models. J. Acad. Mark. Sci. 1988, 16, 74-94. [CrossRef]

56. Nunnally, J.; Bernstein, I. Psychometric Theory, 3rd ed.; McGraw-Hill: New York, NY, USA, 1994.

57. Fornell, C.; Larcker, D.F. Evaluating structural equation models with unobservable variables and measurement error. J. Mark. Res. 1981, 18, 39-50. [CrossRef]

58. Hair, J.F.; Sarstedt, M.; Ringle, C.M.; Mena, J.A. An assessment of the use of partial least squares structural equation modeling in marketing research. J. Acad. Mark. Sci. 2012, 40, 414-433. [CrossRef]

59. Chin, W.W. The partial least squares approach to structural equation modeling. Mod. Methods Bus. Res. 1998, 295, 295-336. 
60. Akter, S.; D'Ambra, J.; Ray, P. An evaluation of PLS based complex models: The roles of power analysis, predictive relevance and GoF index. In Proceedings of the A Renaissance of Information Technology for Sustainability and Global Competitiveness, 17th Americas Conference on Information Systems (AMCIS 2011), Detroit, MI, USA, 4-8 August 2011.

61. Lleras, C. Path analysis. In Encyclopedia of Social Measurement; Elsevier: Philadelphia, PA, USA, 2005.

62. Cohen, J. Statistical Power Analysis for the Behavioral Sciences, 2nd ed.; Lawrence Erlbaum Associates Publishers: Mahwah, NJ, USA, 1988.

63. Cohen, J.; Cohen, P.; West, S.G.; Aiken, L.S. Applied Multiple Regression/Correlation Analysis for the Behavioral Sciences; Routledge: Abington, UK, 2013.

64. Aibinu, A.A.; Al-Lawati, A.M. Using PLS-SEM technique to model construction organizations' willingness to participate in e-bidding. Autom. Constr. 2010, 19, 714-724. [CrossRef]

65. Davison, A.; Hinkley, D. Bootstrap Methods and Their Application; Cambridge University Press: Cambridge, UK, 1997.

66. Efron, B.; Tibshirani, R.J. An Introduction to the Bootstrap. In Monographs on Statistics and Applied Probability Series; CRC Press: Boca raton, FL, USA, 1994; Volume 57.

67. Hair, J.F., Jr.; Hult, G.T.M.; Ringle, C.; Sarstedt, M. A Primer on Partial Least Squares Structural Equation Modeling (PLS-SEM); Sage Publications: Saunders Oaks, CA, USA, 2016.

68. Preacher, K.J.; Hayes, A.F. Asymptotic and resampling strategies for assessing and comparing indirect effects in multiple mediator models. Behav. Res. Methods 2008, 40, 879-891. [CrossRef] [PubMed]

69. Nitzl, C.; Roldan, J.L.; Cepeda, G. Mediation analysis in partial least squares path modeling: Helping researchers discuss more sophisticated models. Ind. Manag. Data Syst. 2016, 116, 1849-1864. [CrossRef]

70. Haas, M.R.; Park, S. To share or not to share? Professional norms, reference groups, and information withholding among life scientists. Organ. Sci. 2010, 21, 873-891. [CrossRef]

71. Hu, M.-L.M.; Horng, J.-S.; Sun, Y.-H.C. Hospitality teams: Knowledge sharing and service innovation performance. Tour. Manag. 2009, 30, 41-50.

72. Černe, M.; Nerstad, C.G.; Dysvik, A.; Škerlavaj, M. What goes around comes around: Knowledge hiding, perceived motivational climate, and creativity. Acad. Manag. J. 2014, 57, 172-192. [CrossRef]

(C) 2019 by the authors. Licensee MDPI, Basel, Switzerland. This article is an open access article distributed under the terms and conditions of the Creative Commons Attribution (CC BY) license (http://creativecommons.org/licenses/by/4.0/). 